\title{
Continuous Heart Rate Monitoring System as an IoT edge device
}

\author{
Johan Bhurny Bathilde ${ }^{1}$, Yi Lung Then ${ }^{2 *}$, Rajith Chameera ${ }^{3}$, Fei Siang Tay ${ }^{4}$, and Dyg Norkhairunnisa Abang Zaidel ${ }^{5}$ \\ ${ }^{1,2,3}$ Department of Electrical and Electronic Engineering, Faculty of Engineering, Computing and Science, Swinburne \\ University of Technology, Sarawak Campus, 93350 Kuching, Malaysia. \\ ${ }^{4}$ Department of Robotics and Mechatronics Engineering, Faculty of Engineering, Computing and Science, Swinburne \\ University of Technology, Sarawak Campus, 93350 Kuching, Malaysia. \\ ${ }^{5}$ Department of Electrical and Electronics Engineering, Faculty of Electrical Engineering, Universiti Malaysia Sarawak, 94300, \\ Kota Samarahan, Sarawak. \\ *Corresponding author ythen@swinburne.edu.my
}

\begin{abstract}
Detection of atrial fibrillation is done by checking the variations in the period of the heart rate, if a patient has atrial fibrillation then the period between each heart beat will vary. A light-based sensor can be used to detect these variations in heart rate; this is done by using Photoplethysmography (PPG) sensor which is non-invasive. The sensor consists of a LED with a photodetector and is able to detect the variations in blood volume or blood flow in the body and directly correlates to heart rate. The detected signal needs to be amplified and filtered as the signal contains a lot of high frequency noise as well as low frequency motion artifacts. The benefits of compact low-cost Wi-Fi module can be harnessed to develop a wireless continuous heart rate monitoring system enhancing possibility of atrial fibrillation detection.
\end{abstract}

Keywords- Light-based sensor, amplification, arrhytmia, filtering, Photoplethysmography, IoT, Wi-Fi.

\section{INTRODUCTION}

Photoplethysmography refers to the non-invasive measurement of blood volume in a specified region (wrist). The volume of blood in a specified region increases in the systole phase and decreases in the diastole phase during the cardiac cycle of the heart. This changing blood volume can be directly used to calculate the heart rate and also to measure other characteristics of cardiovascular functions. It uses LEDs and their corresponding sensor as pairs in retrieving information in the form of electrical signals which a low in amplitude. This electrical signal known as the blood volume pulse signal has to be filtered from high frequency noise sources such as sunlight and ambient light, the signal also contains low frequency noise in the form of motion artifacts. The high frequency noise can be eliminated from the signal completely with the help of an active low pass filter. Most of the low frequency noise contributed by the motion artifacts can be eliminated with the use of a passive high pass filter. The problem with motion artifacts is that they overlap with the blood volume pulse and is difficult to get rid of completely. To get rid of the motion artifacts completely more complex methods will have to implement such as Discrete Saturation Transform, Adaptive Noise Cancellation etc. This project will not try to completely get rid of motion artifacts as this is an on-going research area which has not yet found a comprehensive solution as yet. After the filtering and amplification stage the signal is fed to a microcontroller where the heart rate will be calculated and the period between heart beats will be analyzed to determine the presence of atrial fibrillation. Interfacing the controller with a $\mathrm{Wi}-\mathrm{Fi}$ module allows the transfer of the measured PPG signal and heart rate provided an access point (AP) with internet. A vast number of physical object are given internet access using a small and low power Wi-Fi module hence realizing the concept of Internet of Things (IoT) which refers to the interconnection of "things" and can integrate wireless sensor network with the internet [12]. The application of IoT in healthcare can provide a consequent improvement in healthcare by exploiting its wireless nature as well as the mostly preferred cloud computing and analytics offered by the IoT platform.

An alternative to this method is the photoplethysmographic (PPG) technique used in existing pulse-oximeters. A none invasive method of continuous heart rate monitoring coupled with internet access via a Wi-Fi module allows the wireless monitoring annexed with the cloud computing platform of IoT can offer the possibility of detecting atrial fibrillation for the PPG sensor wearers in the healthcare context where it is assumed little movement during the measuring period in an initial phase.

\section{Methodology}

\section{A. Heart rate measurement}

Photoplethysmography is a method used to determine and register the variations in blood volume or blood flow in the body. This is achieved by directing light to the surface of the skin (finger, wrist) which is absorbed differently by various bodies like pigments in the skin, bone and arterial and venous blood [1]. The arterioles and arteries is where the changes in blood flow are significant. For instance, during the systolic phase the arteries contain more blood volume than the diastolic phase. Therefore, the amount of light absorbed by the blood during the systolic phase is higher than in the diastolic phase, PPG sensors optically detect these variations, by 